Revue internationale P.M.E.

Économie et gestion de la petite et moyenne entreprise

\title{
La marque dans la marque: interorganisation et dynamique de réputation positive pour les entreprises innovantes en BtoB
}

\section{Paul Bouvier-Patron}

Volume 16, numéro 1, 2003

URI : https://id.erudit.org/iderudit/1008433ar

DOI : https://doi.org/10.7202/1008433ar

Aller au sommaire du numéro

Éditeur(s)

Presses de l’Université du Québec

ISSN

0776-5436 (imprimé)

1918-9699 (numérique)

Découvrir la revue

Citer cette note

Bouvier-Patron, P. (2003). La marque dans la marque: interorganisation et dynamique de réputation positive pour les entreprises innovantes en BtoB. Revue internationale P.M.E., 16(1), 75-104. https://doi.org/10.7202/1008433ar
Résumé de l'article

L'objectif affirmé ici est de chercher à appréhender de nouvelles stratégies d'entreprises qui obligent à repositionner la question de la marque dans un univers économique où les frontières entre les entreprises deviennent de plus en plus floues et où la complémentarité d 'activités interentreprises joue un rôle essentiel. La cible visée est donnée par le titre : examiner dans quelle mesure il est valorisant et profitable d'incorporer d'autre(s) marque(s) dans sa marque. Cette question est donc aussi cruciale pour les PME produisant des ingrédients appréciés par des clients. 


\title{
Notes de recherche
}

\section{La marque dans la marque: interorganisation et dynamique de réputation positive pour les entreprises innovantes en BtoB}

\author{
Paul BOUVIER-PATRON \\ Qualité et efficacité des organisations et des processus (QEOPS), \\ Université de Toulon et du Var \\ Innovation système stratégie (I.SY.S.), \\ Université de Paris I
}

\section{MOTS CLÉS}

\author{
BtoB - Facteurs clés de succès - Firme \\ Interorganisation - Stratégies de marque
}

\begin{abstract}
RÉSUMÉ
L'objectif affirmé ici est de chercher à appréhender de nouvelles stratégies d'entreprises qui obligent à repositionner la question de la marque dans un univers économique où les frontières entre les entreprises deviennent de plus en plus floues et où la complémentarité d'activités interentreprises joue un rôle essentiel. La cible visée est donnée par le titre : examiner dans quelle mesure il est valorisant et profitable d'incorporer d'autre(s) marque(s) dans sa marque. Cette question est donc aussi cruciale pour les PME produisant des ingrédients appréciés par des clients.
\end{abstract}

\section{L'AUTEUR}

PaUl Bouvier-Patron est spécialiste d'économie et de stratégie industrielle. Ses travaux ont pour axe principal l'analyse de la qualité des relations clients-fournisseurs où l'élaboration de la réputation (la stratégie de marque en étant un vecteur) apparaît comme un phénomène essentiel. Adresses : Université de Toulon et du Var, QEOPS, B.P. 132, 83957 La Garde cedex, France et I.SY.S., Université de Paris I Panthéon-Sorbonne, MSE, 106, boul. de I'Hôpital, 75013 Paris, France. 


\begin{abstract}
The aim of this paper is to take into account new strategies of firms which put into question branding in an economic world where borderlines between firms, on a practical point of view, tend to disappear and where complementarity of activities between firms is essential. The target is explained by the title: that is to say, to examine in which circumstances it is a good thing to incorporate other(s) brand(s) in its own brand. In the context of buyers-sellers relationships, this is a considerable stake for small and medium firms.

\section{RESUMEN}

Intentaremos aprehender nuevas strategias de impresas que obligan a volver a positionar la questiòn de la marca en un universo econòmico donde las fronteras entre empresas son cada vez màs borrosas y donde la complementariedad de actividades inter-empresas desempeña un pàpel esencial. El objetivo que nos hemos fixado esta totalmente traducido por el titulo : examinar en que medida puede ser valoizante y provechoso incomporar otra(s) marca(s) en su marca. Esta questiòn es crucial para las PyMEs que realizan ingredientes y que son muy implicadas en las relationes clientes-proveedores.
\end{abstract}

\title{
ZUSAMMENFASSUNG
}

Das behauptete Ziel ist hier nach neuen Unternehmensstrategien zu suchen, die dazu führen, die Frage der Marke in einem wirtschaftlichen Umfeld neu zu positionieren, bei dem die Grenzen zwischen den Unternehmen mehr und mehr verwischt werden und die Ergänzung der zwischenbetrieblichen Aktivitäten eine wichtige Rolle spielen. Die anvisierte Zielgruppe ist gegeben durch den Titel : Prüfen in welchem Ausmass es wertbildend und vorteilhaft ist, andere Marken in seine eigene Marke einzuverleiben. Diese Frage ist höchstwichtig für die kleinen und mittleren Unternehmen, welche von den Kunden geschätzte kleine Bestandteile abdecken.

\section{Introduction}

Si la marque est un enjeu de plus en plus important, c'est que, pour une entreprise, le réflexe de déposer un brevet n'apporte qu'une sécurité limitée (indispensable mais non suffisante), car bien vite dépassée pour parvenir à contrer l'imitation des concurrents ou même, préalablement, pour retenir l'attention du client (souvent rétif à s'engager a priori et, généralement, désireux d'être rassuré pour ce faire). Il faut donc bien tabler sur une valeur intangible qui produit un signal durable, mémorisé par le client actuel et potentiel, concernant les produits existants ou futurs : la marque est ce signal ${ }^{1}$. Concomitamment, les entreprises se situant dans

1. Sauf à dire que le produit pris comme combinaison de caractéristiques techniques suffit pour générer une vente ou encore que la satisfaction client n'est pas primordiale.

Revue internationale P.M.E., vol. 16, no 1, 2003

(C) 2003 - Presses de l'Université du Québec

Édifice Le Delta I, 2875, boul. Laurier, bureau 450, Sainte-Foy, Québec G1V 2M2 • Tél. : (418) 657-4399 - www.puq.uquebec.ca

Tiré de : Revue internationale P.M.E., vol. 16, $\mathrm{n}^{\circ} 1$, sous la direction de Pierre-André Julien. 
une chaîne de valeur (qui, nécessairement aujourd'hui, déborde leurs frontières internes, $c f$. infra) se posent la question du choix d'éventuelles combinaisons de marques: d'où l'interrogation proposée ici sur la marque dans la marque. Autrement dit, le choix de défendre sa propre marque seul ou, au contraire, à travers une combinaison de marques tant que ceci correspond bien à une solution adaptée au contexte concurrentiel et aux besoins détectés. Quoi qu'il en soit, dans tous les cas, l'objectif stratégique amont des entreprises consistera à s'appuyer sur différents facteurs clés de succès : $i$ ) la complémentarité de compétences interentreprises, la construction ii) d'une barrière à la mobilité durable mais iii) sans se spécialiser trop fortement tout en recherchant, progressivement, en aval, $i v$ ) la capture et la canalisation d'une demande devenue «addictive»; la réussite ultime, en effet, étant de convaincre le client du client pour asseoir la légitimité définitive d'une marque d'ingrédient. C'est donc, par exemple, en analysant son potentiel stratégique et en repérant des acteurs liés complémentaires que les petites et moyennes entreprises (PME) peuvent se situer dans un espace de valorisation leur permettant de tirer leur épingle du jeu.

Ainsi, la marque (indépendamment du brevet) constitue un enjeu fondamental pour une entreprise désireuse de croître ; surtout si celle-ci a l'ambition d'asseoir une image lui permettant de s'affranchir d'une niche de départ trop étroite. En même temps, la réussite d'une telle politique (qui se décline en plusieurs stratégies possibles) va dépendre du contexte concurrentiel et d'un certain nombre de facteurs clés de succès que nous nous proposons d'examiner ici. Cela dit, la généralisation envisagée pose le problème de l'extension de l'analyse à la PME dans la mesure où il a souvent été postulé que la taille de l'entreprise devait être considérée comme un déterminant fondamental : une petite taille signifiant nécessairement dépendance. Or, nous avons déjà cherché à montrer (Bouvier-Patron, 1996) que « les PME ne sont pas intrinsèquement dominées du fait de leur taille mais, essentiellement, quand c'est le cas, par l'absence de compétences reconnues ». Ainsi, selon les compétences acquises ou en construction, la PME dispose donc, ou pas, d'un pouvoir de négociation (Porter, 1980, 1985; Aoki, 1988; Asanuma, 1989; Bouvier-Patron, 1994, 1996, 1998a, 1998b). Ce pouvoir de négociation transcende la dépendance, généralement admise, de la PME (particulièrement, dans le cadre qui nous intéresse ici, en tant que fournisseur de clients que sont les grandes entreprises) en permettant d'équilibrer une relation.

Nous allons ainsi chercher à appréhender de nouvelles stratégies d' entreprises qui, utilisant ce pouvoir de négociation, obligent de fait à repositionner la question de la marque dans un univers économique où, par ailleurs, les frontières entre les entreprises deviennent de plus en plus floues et où la PME peut jouer un rôle considérable en tant que support éventuel d'une marque d'ingrédient(s). Sur le plan le plus général, la question fondamentale posée revient donc à examiner dans quelle mesure il est valorisant et profitable d'incorporer d'autre(s) marque(s) dans

Revue internationale P.M.E., vol. 16, n 1,2003 
sa marque. Pour y répondre, dans le cadre des relations clients-fournisseurs, nous chercherons à proposer une grille d'analyse systématique ( $c f$. conclusion) fondée sur des idéaux-types à portée théorique et pratique visant à révéler les potentialités des modalités relationnelles et des logiques associées en termes de marquage. Il s'agit, pour ce faire, de marier des travaux d'origines diverses à l'intersection du marketing industriel et de l'économie industrielle.

Par convention, nous prendrons généralement le point de vue de l'intégrateur d'ingrédients (firme pivot), car cela offre la possibilité de traiter le problème sous l'angle le plus général; positionnement que nous préserverons le plus possible même si notre objectif, dans le cadre de la relation client-fournisseur, est bien de valoriser l'action du fabricant d'ingrédient(s) qui est, généralement, une PME. De ce fait, on peut circonscrire notre champ d'étude à deux éclairages complémentaires : le premier éclairage concerne le point de vue adopté selon les deux angles client et fournisseur (distincts mais non nécessairement contradictoires); le second éclairage consiste à envisager les facteurs clés de succès relatifs à chaque choix stratégique possible. Autrement dit, au total, on obtient respectivement un double éclairage :

- Quels sont les choix, en matière de politique de marque, pour un industriel en tant qu'utilisateur ou fabricant d'ingrédient(s)?

- Quels sont les facteurs clés de succès (liés à chaque choix stratégique possible) au sein de la politique de marque?

Pour répondre à ces deux questions, nous envisagerons d'abord ( $c f$. 1.) la tendance lourde de l'organisation industrielle contemporaine fondée sur la complémentarité d'activités interfirmes (Richardson, 1972) : celle-ci explique le développement croissant et irréversible du phénomène, encore marginalisé pourtant, qualifié ici de « la marque dans la marque ». Nous présenterons ensuite ( $c f .2$.) comment le flou des frontières de la firme, lié à cette mutation profonde de l'organisation industrielle (dont un des corollaires est la montée en puissance de la fonction achat) induit la nécessité de combinaisons de marques fortes ${ }^{2}$. Dès lors ( $c f$. 3.), nous aborderons les conséquences engendrées au niveau de la perception du client. Nous verrons ainsi que l'acte d'achat, intimement lié au jugement, implique une convergence sur telle marque si celle-ci est gage de confiance : par extension, la convergence des clients particuliers que sont les acheteurs vers telle combinaison de marques traduit un gage de confiance. Cette confiance semble reposer d'ailleurs

2. La définition de marque forte donnée par Kapferer (1998, p. 62) est la suivante : «la marque forte est celle qui distribue ses valeurs et parvient à segmenter le marché selon ses propres normes. Elle cherche à imposer son propre standard ».

Revue internationale P.M.E., vol. 16, nº 1, 2003 
sur une certaine inertie du fait, notamment, de l'attachement au premier entré ${ }^{3}$. Enfin, en conclusion, nous synthétiserons différentes stratégies possibles et leurs facteurs clés de succès associés de façon à déboucher sur une grille d'analyse globale.

\section{La logique de complémentarité}

L'entreprise, centrée sur un métier, détient des compétences (technologiques et / ou de savoir-faire) grâce auxquelles elle élabore, réalise puis vend des produits. Imaginons que cette entreprise existe déjà, elle a dû investir en communication, directement et indirectement, auprès de ses clients (c'est-à-dire, ici, d'autres entreprises $\left.{ }^{4}\right)$ :

- en approche directe, lors de visites ou de salons ;

- en publicité sur ses produits (depuis la distribution de catalogues jusqu'au film publicitaire en passant aussi, aujourd'hui, par des bandeaux publicitaires sur Internet);

- sur le plan institutionnel afin de signifier son identité et valoriser son image, voire peut-être de corriger cette image (en interne mais aussi à destination de l'extérieur);

- par la valorisation de sa marque qui est son emblème (en tant que synthétisation des produits en même temps que synthétisation de l'entreprise) et perçu comme tel dans l'imaginaire individuel et collectif en interne et, surtout, en externe.

Or donc, pour communiquer, la marque occupe une place de plus en plus essentielle dans la stratégie d'entreprise dans la mesure où elle est, dans tous les cas, outre un capital important ( $c f$. Kapferer, 1998), «l'image de marque » de l'entreprise. Par la marque, il s'agit de véhiculer un signal auprès des clients : un signal perceptible par les clients pour différentes raisons (prestige, culture, confort, sécurité, déculpabilisation d'achat, signe de qualité5). L'entreprise émet ainsi un signal fort, lequel est porté par ses produits, concernant sa culture, ses compétences, son potentiel technologique et son savoir-faire.

3. Kapferer (1998, p. 160-161) : «L'explication psychologique tient à ce que, à la naissance d'un marché, les acheteurs n'ont pas de système de préférences, pas de critères de choix stabilisés. La première marque parvenant à être connue dans ce marché en devient le prototype, le référent. »

4. Rappelons que ce sont les relations « $\mathrm{BtoB} »$ qui vont nous intéresser ici.

5. La mise en place de labels dans l'agroalimentaire est la réponse institutionnelle, en liaison avec les professionnels, au problème soulevé par Akerlof (1970) concernant l'incertitude sur la qualité des produits. 
L'objectif est, grâce à une panoplie de moyens, d'asseoir un effet de réputation ( $c f$. Selten, 1978; Kreps et Wilson, 1982; Axelrod, 1984) de l'entreprise pour que l'acte d'achat des clients potentiels se porte sur le produit marqué. Par simplicité, réputation et notoriété seront ici synonymes: d'habitude, la réputation joue fortement dans le seul cadre professionnel (en BtoB) et la notoriété, comme un indicateur mesurable permettant d'appréhender la popularité d'un nom, d'un produit ou d'une prestation proposée auprès des clients (en BtoB ou en BtoC).

Selon nous, la logique de réputation retenue ici comme principe général est assise, conceptuellement et pratiquement, sur une valorisation réussie des compétences appartenant au cœur de métier d'une entreprise.

Toutes les entreprises ne disposent pas ou n'utilisent pas de tels moyens pour parvenir à vendre leurs produits ou, plus globalement, leurs prestations. En effet, des entreprises survivent et / ou vivent sans nécessairement se faire connaître publiquement. Aussi, nous parlions bien de la marque comme emblème et non comme quintessence de l'entreprise, car de nombreuses entreprises, petites et moyennes surtout, existent aussi et se développent sans pour autant communiquer sur leurs produits ou leurs savoir-faire. Elles travaillent alors anonymement (du point de vue du consommateur final) pour le compte d'autres (et donc, sous la marque de ces dernières); c'est particulièrement vrai dans le secteur de la grande distribution avec les marques de distributeurs (MDD) concurrençant les marques nationales.

Toutefois, il faut aussi noter que les MDD de dernière génération ne sont plus des copies mais bien des innovations apportant parfois une valeur supérieure aux marques nationales; les fabricants de ces MDD étant aussi parfois les usines des marques nationales elles-mêmes (afin de maintenir leurs débouchés). La référence aux MDD de dernière génération est importante ici puisque, comme le soulignent Malaval et Bénaroya (1998, p. 63), les distributeurs cherchent, désormais, à combiner leurs marques de distributeurs aux marques des fabricants-fournisseurs : « la juxtaposition de la marque du distributeur à celle du fabricant correspond à la volonté du distributeur industriel d'additionner les avantages procurés par l'image de marque du fabricant et ceux de sa propre image »; ainsi, la marque du fabricant cautionne le bien lui-même tandis que la marque du distributeur cautionne le service apporté autour du produit.

C'est pourquoi il faut bien aussi considérer la logique - pour le compte d'autres - puisque, dans une vie des affaires mouvantes où l'hyper-concurrence sévit, ces «autres» sont, en fait, la majorité des entreprises recentrées sur leur métier et qui délèguent à d'autres tout ou partie de la réalisation de leur produit (et/ou service), pour des motifs variés et, parfois, cumulatifs de recherche de moindres coûts ou de savoir-faire non maîtrisés en interne et / ou trop onéreux à maîtriser. L'enjeu, aujourd' hui, est de savoir si les intermédiaires, apportant les 
"ingrédients », ont intérêt à une visibilité dans le produit final et si cette visibilité doit porter le sceau d'une marque (sachant donc que toutes les entreprises ne défendent pas nécessairement une marque).

Ainsi, la réalité de l'organisation industrielle n'étant pas binaire, il n'y a pas que des logiques duales entre entreprises. Prenons deux exemples de dualités fortes:

- entreprises réalisant l'ensemble des compétences nécessaires à l'obtention du produit (ce qui est de plus en plus rare aujourd'hui) versus celles qui travaillent avec d'autres;

- entreprises disposant de leur marque propre versus celles travaillant pour le compte d'autres.

En fait, on trouve de plus en plus d'entreprises en interaction avec d'autres développant des stratégies hybrides où, simultanément, pour partie, elles disposent de produits vendus sous leur marque et, pour partie, des produits (ou parties de produits) réalisés pour le compte d'autres.

À ce stade, ce qu'il importe de noter est que les stratégies de délégation s'imposent (tactiquement parfois ou stratégiquement le plus souvent), ici aussi, pour des raisons de complémentarité: en termes de capacité (pour trouver les capacités de production manquantes en interne); le plus souvent en termes de spécialité (afin d'obtenir, à de meilleurs coûts, l'accès à des compétences technologiques ou de savoir-faire non maîtrisés et parfois rares en interne), ce qui est propice à l'innovation.

Ce recours à la complémentarité d'activités (Richardson, 1972) a pris une ampleur considérable depuis les années 1980 compte tenu des stratégies de recentrage sur le métier (Teece, 1980, 1988) et, corrélativement, d'externalisation (avec donc le recours à des «spécialistes » complémentaires à la firme que sont les fournisseurs). Cette tendance de fond a souvent été occultée par des phénomènes plus spectaculaires comme les fusions d'entreprises (rendues nécessaires par la globalisation des marchés et la mondialisation de l'économie). Dans tous les cas, les mouvements économiques majeurs précités (recentrage, externalisation, fusion, globalisation, mondialisation, etc.) ont des incidences sur les entreprises et, en particulier, sur les marques.

Nous nous intéresserons ici moins au côté spectaculaire (fusions, etc.) qu'à ce qui s'impose véritablement, concrètement, immédiatement et durablement, sur le plan industriel et commercial : les entreprises ne peuvent plus compter sur ellesmêmes exclusivement et doivent nouer des liens avec d' autres pour des raisons de complémentarité. Il faut alors étudier à quelles conditions cette tendance de fond a des conséquences sur la marque, et ce, alors que plusieurs scénarios sont envisageables:

Revue internationale P.M.E., vol. 16, $\mathrm{n}^{\circ}$ 1, 2003 
- n'utiliser et ne défendre que la marque de l'entreprise désireuse de vendre le produit indépendamment de l'existence d'ingrédients (ingrédients non réalisés en interne), marqués ou pas, constitutifs du produit;

- utiliser la marque du produit vendu mais sans possibilité de dissimuler les marques des ingrédients (parce que ces dernières sont réclamées par le consommateur ou bien s'imposent par l'existence de monopoles ou encore par des exigences techniques);

- chercher à imposer la marque de l'entreprise vendant le produit final à l'aide des marques constitutives des ingrédients : soit de façon explicite, soit de façon implicite (et, dans ce dernier cas, utilisation parfois maligne ou de détour d'image avec les problèmes juridiques que cela peut entraîner).

Si on laisse de côté le cas d'une stratégie de prédation visant à détourner l'image d'autres marques concurrentes et/ou de marques d'ingrédients (une telle analyse valant surtout pour l'intérêt des débats juridiques autour de la question de la propriété intellectuelle), on obtient bien, au total, plusieurs tendances stratégiques possibles:

a) défendre sa marque seul en n'utilisant que des ingrédients que l'on rend insignifiants ;

b) défendre sa marque tout en faisant appel à d'autres réputés qui travaillent « silencieusement » pour le compte de cette marque (comme fournisseurs ou partenaires);

c) défendre sa marque en recourant explicitement à d'autres (marques complémentaires);

d) défendre une marque commune et de façon conjointe par des entreprises ayant des activités similaires généralement ou bien associées ponctuellement pour une opération commerciale.

Naturellement, ces stratégies peuvent, globalement, coexister au niveau du portefeuille d'achats de l'entreprise cliente selon la politique choisie par produit. Par ailleurs, on peut présenter l'analyse des différentes stratégies à l'aide de deux axes :

- sur un axe vertical, regroupant potentiellement toutes ces stratégies $(b, c$, d) mais selon un degré d'interaction partenariale interentreprises plus ou moins affirmé, où la dimension de complémentarité prévaut;

- sur un axe horizontal, correspondant à certaines situations, au sein de la stratégie $(d)$, où l'alliance conclue est, finalement, plutôt tactique à court terme ainsi que commerciale.

Revue internationale P.M.E., vol. 16, nº 1, 2003 
Ainsi, la complexité qui existe (aujourd'hui plus encore) nous oblige à dépasser les perceptions habituelles de la marque puisque cette perception est devenue fluctuante dans la mesure même où les frontières des entreprises fluctuent également (par une distorsion entre les droits de propriété concernant les frontières de l'entreprise et les réalités organisationnelles et stratégiques telles que nous venons de les évoquer). En réalité, la propriété existe toujours mais ne suffit plus à définir le champ pertinent d'action sur le plan de l'élaboration et de la réalisation des produits ; les interactions consécutives à la création, la gestion, le développement des complémentarités conduisant à la prise en compte d'une métaorganisation, de méta-actions, voire peut-être, également, la constitution de métamarques.

Dans le même temps, ces réalités organisationnelles et stratégiques actuelles sont explorées de façon assez parcellaire (lorsqu'elles le sont) et il nous semble intéressant d'essayer de proposer une approche unifiée permettant de donner un cadrage explicatif général.

\section{Des frontières floues de l'entreprise aux marques fortes}

Rao et Rueckert (1994) ont donné un premier cadrage très pertinent et ont insisté sur l'alliance de marques (au sens de la stratégie (c) ici). Kapferer (2000) a abordé aussi, à l'opposé, la question de la fusion de marques (consécutive aux processus d'acquisitions qui sont, à titre direct, hors de notre champ d'étude) tandis que Kapferer (1998) et Malaval (1996, 1998) ont proposé un éclairage sur les marques d'ingrédients ou marques incorporées (terminologie utilisée, respectivement, par ces deux auteurs).

On voit que le spectre balayé est assez large et qu'il est important de chercher à en produire une vue plus synthétique quant aux aspects déterminants. D'autant plus que les stratégies suivies par les entreprises recouvrent des réalités différentes : une même entreprise peut chercher à défendre certains produits sous sa propre marque, et ce, tout en travaillant pour d'autres et / ou avec d'autres (comme fournisseurs de premier rang ou, simplement, comme sous-traitant); parfois aussi, en collaboration pour élaborer des marques conjointes et, éventuellement même, travailler à des marques collectives (avec ou sans aval institutionnel).

La correspondance (implicitement évoquée) entre les stratégies d'achat (grosso modo : faire seul, faire-faire, faire avec d'autres) et les possibles positionnements de marque évoqués est une résultante des bouleversements profonds en cours au sein de l'organisation industrielle (notamment avec le développement considérable d'une sous-traitance de qualité, de l'externalisation, du partenariat) alors même qu'une interaction de plus en plus étroite peut aussi exister entre le marketing aval et le marketing amont (trade-marketing et category management pour prendre un exemple évident mais novateur dans le secteur de la distribution alimentaire).

Revue internationale P.M.E., vol. 16, $\mathrm{n}^{\circ}$ 1, 2003

(C) 2003 - Presses de l'Université du Québec

Édifice Le Delta I, 2875, boul. Laurier, bureau 450, Sainte-Foy, Québec G1V 2M2 • Tél. : (418) 657-4399 - www.puq.uquebec.ca

Tiré de : Revue internationale P.M.E., vol. 16, $\mathrm{n}^{\circ} 1$, sous la direction de Pierre-André Julien. 
Nous insisterons surtout sur l'amont pour limiter l'investigation. Par ailleurs, on sait la complexification des logiques en présence à mesure que la cible devient le consommateur final. On s'intéressera à la question du consommateur final dans la mesure surtout où celui-ci est lié à l'usage de biens d'équipements (soit en termes d'acquisition dans le cas de l'automobile par exemple; soit au niveau de l'usage dans le cas d'Internet ou des transports collectifs) : à ce niveau, les logiques en œuvre en «BtoC» (entreprise-consommateur) sont relativement proches (même si non similaires) de celles qui existent en « BtoB » (entre entreprises).

Cette précision faite, il ne faut cependant en rien mêler cet aspect avec une stratégie de capture ( $c f . i$ ) infra) ou, tout au moins, de conditionnement des consommateurs pour servir de levier par répercussion vers l'amont ( $c f$. 2.1. tirée par le marketing) afin d'orienter la demande vers les produits de l'entreprise. Plus généralement, la classification des ingrédients ( $c f .2 .2$.) doit alors être envisagée.

\subsection{Le levier que constitue une certaine politique tirée par le marketing}

L'enjeu central de l'entreprise est la valorisation des compétences du cœur de métier, cette valorisation pouvant s'affirmer par une logique de réputation existante ou à établir dans la mesure où, peu ou prou, une entreprise qui veut croître cherche à étendre son marché. Or, pour étendre son marché, elle doit sortir de sa niche initiale (où elle a pu asseoir sa réputation) de manière à parvenir à la conquête de plus vastes espaces (en utilisant la réputation).

Le problème des entreprises amont proposant des prestations d'ingrédients à d'autres entreprises est justement que ces premières entreprises sont limitées, au départ, à exister dans une filière (verticale) au sein d'une niche initiale. Pour en sortir, la première réponse qu'est la multiplication des clients (et, dans une moindre mesure, aujourd'hui, du fait du recentrage sur le métier, la diversification des activités) ne constitue pas un espace de croissance suffisant, surtout pour une entreprise ambitieuse et appréciée pour ses prestations. Cette entreprise peut donc, en deuxième réponse, chercher à s'évader de sa niche en court-circuitant les intermédiaires ne serait-ce que par une présence dans l'imaginaire de tout un chacun en aval. Cet objectif passe par une communication directe auprès du consommateur final: l'entreprise concernée doit convaincre le consommateur final que, derrière un produit final donné contenant, implicitement ou explicitement, l'ingrédient (fourni par elle), cet ingrédient représente une valeur additionnelle (parfois presque toute la valeur).

On peut identifier Gore-Tex, Intel, Lycra, Shimano comme des entreprises ayant réussi ce type de stratégie ambitieuse : i) l'enjeu étant de conditionner le consommateur pour que celui-ci n'achète telle catégorie de produit qu'à condition que l'ingrédient de marque soit explicitement présent à l'exclusion de tout autre 
équivalent. Ce conditionnement du consommateur final oriente les achats de celui-ci et fait remonter l'impulsion dans le canal vertical amont jusqu'à obliger les intermédiaires (distributeurs et/ou producteurs) à s'adresser exclusivement (ou presque) à l'entreprise émettrice du signal fort ainsi construit.

On ne peut douter que parvenir, en aval, à un tel contrôle sur l'acte d'achat repose bien, en amont, sur une valeur intrinsèque forte du produit vendu ; la qualité du produit concerné étant avérée, en particulier, du fait des compétences technologiques ou de savoir-faire incorporés.

Néanmoins, dans un univers de compétition exacerbée et alors que la course à l'innovation est permanente ainsi que les cycles de vie des produits de plus en plus courts, il n'est pas dit que les solutions proposées par la concurrence (à plus ou moins brève échéance) ne soient pas équivalentes : donc, pas nécessairement pires a priori mais pourtant bien délaissées par le consommateur lorsqu'il n'a pas été sollicité ou qu'il n'a pas été convaincu par les signaux envoyés par les entreprises apportant des solutions pensées comme équivalentes. Un cas connu, même s'il n'a pas fait l'objet d'une vérification précise, comme l'entreprise Salomon qui, ayant développé sa propre fibre, est cependant contrainte d'utiliser celle de Gore Tex pour réaliser des produits trouvant des acheteurs.

À l'évidence, ces solutions concurrentes sont donc faiblement équivalentes du point de vue de la valeur puisqu'elles ne sont pas désirées de la même façon. On voit donc que la question de la réputation de la marque, grâce au potentiel de l'entreprise (via ses compétences, sa technologie ou son savoir-faire) est une chose primordiale, mais que l'art de communiquer sur ce potentiel (agissant sur la perception de la marque) l'est tout autant.

Un élément explicatif clé devrait être mis en avant: ii) la réputation du premier entré, ou supposé tel, semble déterminante et cela suggère que le consommateur associe l'authenticité au premier arrivé. Le premier arrivé apparaît ainsi comme le premier à créer un lien spécifique entre sa marque et le consommateur (et donc non pas nécessairement le premier à avoir innové technologiquement). L'objectif est alors non seulement d'être le premier à créer ce lien, mais aussi à maintenir son avance dans le temps.

Il y a donc une gradation importante qui peut être, ou non, couverte par une seule et même entreprise (ce qui n'est pas nécessairement rare mais très difficile) :

- être le premier à innover technologiquement et à créer un type de produit nouveau;

- être le premier à faire connaître ce type de produit ;

- être le premier à créer un lien spécifique fort entre une marque et le consommateur;

- être le premier à créer ce lien et à préserver cet avantage dans le temps.

Revue internationale P.M.E., vol. 16, n 1, 2003 
Il est donc assez important de nuancer le concept de «premier entré » selon qu'on se place du point de vue technologique ou commercial : ainsi, Lycra n'est pas la première marque concernant une fibre élastique et confortable inventée, mais elle a été, commercialement, la première à s'imposer ainsi que la première, voire la seule, à être reconnue par les clients (d'où l'importance de la formation du jugement, $c f .3$.).

Les probabilités de succès de vente seront d'autant plus élevées que l'acheteur aura une croyance d'un lien fort entre une performance d'un attribut et la marque et que ce lien sera exclusif (grâce à une visibilité comme première marque entrée commercialement par exemple, et ce, sans nécessairement donc être la première entrée technologiquement).

La préemption, au sens des «avantages d'être le premier» (connu par la théorie des jeux), consistant ici à réussir à imposer, le premier, un produit et / ou une marque est un atout essentiel (en termes d'avantages concurrentiels immédiats) et constitue ainsi, probablement, les bases d'une barrière à la mobilité (Caves et Porter, 1977). Barrière dont le contournement n'est envisageable, par d'autres, que dans un terme assez lointain et même parfois toujours différé si le premier entré continue à reconstruire en permanence la barrière et parvient ainsi à préserver son avantage dans le temps.

\subsection{Une classification des marques d'ingrédients}

En nous concentrant sur l'analyse de la marque d'ingrédient, il est important de s'appuyer sur le travail de Malaval (1999, p. 78). Cet auteur dresse une typologie en quatre classes des marques industrielles en fonction des attentes et des craintes des clients...

- Dans une classe 1 se situe Gore Tex, c'est-à-dire une marque appréciée, surtout actuellement pour des motifs de performance commerciale induite plutôt que pour son apport technique ou d'innovation pourtant incontestable (du moins au départ).

- Dans une classe 2 on trouve, au contraire, des marques appréciées sur le plan technique, mais ayant une faible influence sur les prescripteurs en aval (comme Lafarge); c'est probablement dans cette classe que les choix d'ingrédients sont les plus objectifs et répondent le mieux à des besoins de complémentarité tels qu'on a pu les aborder plus haut.

- Dans une classe 3, il y a des marques comme Lycra ou Tetra qui allient une désirabilité technique, un impact commercial et une notoriété insolente au point de supprimer toute marge de manœuvre ou toute solution 
alternative (enfermant ainsi l'acheteur dans un durable lock-in relationnel au sens de Schelling [1956, 1960]); à cette classe «championne» correspond une sorte «d'attractivité fatale ».

- Dans une classe 4 se situent les marques ayant le moins d'effet et ne se caractérisant ni par leur impact commercial, ni par leur apport technique (ni d'ailleurs par une crainte ou peur quelconque de la part du client acheteur).

Généralement, une entreprise désireuse de réaliser un produit est dans l'obligation de recourir à d'autres. Or, si l'on retient cette typologie comme valable, le choix d'ingrédient de marque $X$ par rapport à un autre ingrédient d'une autre marque ne peut être le fruit du hasard. En effet, la décision à prendre $(\rightarrow$ classe d'appartenance de l'ingrédient à choisir) résulte de l'impact escompté basé sur un centrage de valeur:

- un centrage sur la valeur de sa seule marque propre mais nécessitant cependant un recours à des complémentarités pour des raisons techniques $(\rightarrow$ classe 2$)$;

- un centrage de valeur sur sa marque, mais, ici, en s'aidant aussi d'ingrédients liés à des marques techniquement reconnues ne se cannibalisant pas entre elles grâce à leurs bonnes notoriétés respectives ( $\rightarrow$ classe 2$)$;

- un centrage sur au moins deux marques à combiner pour un concept global : approche équilibrée de forces strictement égales ( $\rightarrow$ classe 2 );

- une absence totale de centrage sur la marque du produit final pour une action opportune de marché dans un horizon de court terme ( $\rightarrow$ classe 1$)$ ou bien dans une optique assez strictement commerciale mais s'inscrivant dans la durée ( $\rightarrow$ classe 3 ); dans ce dernier cas, il s'agit de s'appuyer sur des valeurs fortes d'ingrédients comme véritables vecteurs de réussite (même au prix d'un effacement complet de la marque du produit réalisé).

On peut envisager toutes ces situations comme polaires, donc envisager également les situations intermédiaires.

Cette réflexion peut aussi être adaptée à des logiques de gamme et permettre ainsi d'ajuster chaque centrage à chaque produit proposé dans la gamme.

La synthèse utile pour favoriser une extrapolation peut être formalisée par un tableau (tableau 1) croisant un axe technologique (horizontal) de la marque et un axe commercial (vertical), ce qui donne un outil de classement dynamique de la politique de marque d'ingrédient (par l'utilisateur ou le fabricant).

Revue internationale P.M.E., vol. 16, $\mathrm{n}^{\circ}$ 1, 2003 
TABLEAU 1

Matrice de classement des marques industrielles selon deux axes d'action entrepreneuriale sur le produit

Action technologique $\begin{gathered}\text { Limitation à un cahier } \\ \begin{array}{c}\text { Res charges acceptable } \\ \text { pour pouvoir vendre }\end{array}\end{gathered} \quad \begin{gathered}\text { Rermanentes pour conforter } \\ \text { permentendre la part de marché } \\ \text { et étende }\end{gathered}$

Communication forte d'image

en plus des caractéristiques

$\begin{array}{lll}\text { du produit } & \text { Classe } 1 & \text { Classe } 3\end{array}$

Communication faible

et travail sur les seules

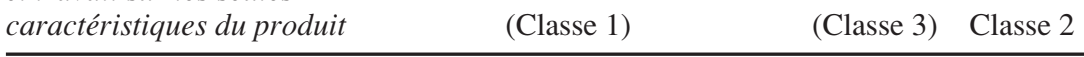

Note : La classe entre parenthèses signale une trace de présence possible sans prédominance.

La «marque dans la marque » prend donc tout son sens lorsqu'on perçoit la complexité des stratégies possibles et que ces possibilités ne sont pas des éventualités théoriques mais des pratiques concrètes (sans qu'existent pour autant jusqu'à aujourd'hui des systèmes explicatifs, même si des aperçus intéressants et pertinents sont parfois proposés).

C'est bien l'identification des stratégies possibles ainsi que celle des facteurs clés de succès pour penser des idéaux-types qui permet de proposer, ensuite, une grille d' analyse utile à l'élaboration d'un guide d' action. En effet, l'optique consiste à présenter les bases conceptuelles avec, dans la mesure du possible, un souci d'être opérationnel pour permettre une prise de décision par les praticiens.

Pour l'instant, nous avons encore à expliquer ( $c f .3$.) pourquoi le consommateur associe l'authenticité au premier arrivé (c'est-à-dire au premier à être reconnu ; puis à préserver le lien spécifique entre marque et consommateur). C'est là le socle de l'une de nos hypothèses importantes: ii) la réputation du premier entré (ou perçu comme tel) semble déterminante.

On peut toutefois déjà noter qu' «être le premier» est dépassable dans la mesure où il est légitime de penser que les compétences détenues ou, en cours d'acquisition, autorisent l'élaboration d'ingrédients performants qui trouveront des débouchés; puis, ces compétences serviront aussi à asseoir, si ce n'est déjà fait, une marque ainsi qu'une réputation durable et enviée ; ce qui peut permettre, enfin, de reconsidérer une situation antérieure monopolistique et de briser le leadership d'un pionnier. 
Pour aller plus loin, on peut aussi penser qu'une réputation stable et la visibilité d'une marque ne sont possibles, à la base, que grâce à iii) la mise en place d'un ensemble de compétences donnant une capacité générique (donc ouverte sur de multiples applications différentes) et rapidement redéployable ${ }^{6}$ sur un nouvel enjeu technologique ou bien permettant des adaptations continues des ingrédients / prestations proposées aux clients.

Par ailleurs, de la logique d'orientation tirée i) de conditionnement du consommateur final, il faut, en outre, mentionner ( $c f$. 1., 2.) l'importance du facteur de complémentarité : $i v$ ) la classe 2 de complémentarité technique offre des garanties pour une véritable stratégie industrielle commune en ouvrant la possibilité de constituer un avantage concurrentiel effectif. Naturellement, la faille de cette dernière vision objective et technique exclusive se trouve dans l'existence de l'incertitude portée par le marché (Knight, 1921). Cette incertitude est liée, en fait, à la difficulté de prévoir les actes d'achats futurs des clients potentiels. Et c'est là tout ce qui fonde la légitimité du recours à l'approche marketing.

C'est pourquoi il est fondamental de ne pas se placer exclusivement du point de vue du fabricant de l'ingrédient, ou de l'ensemblier qui incorpore les ingrédients, mais aussi de celui du client intermédiaire et final.

\section{Jugement du client face aux marques et à la combinaison de marques}

Dans un premier temps ( $c f .1$.$) , nous avons dressé une taxonomie des stratégies$ (ou choix possibles) et étudié ( $c f .2$.), sans prétendre à l'exhaustivité, les facteurs clés de succès afin de prédire la réussite d'une stratégie. Ce qui devient alors crucial est de parvenir ( $c f .3$.) à intégrer le jugement des clients (entreprises et consommateurs) sur les marques (et donc aussi les combinaisons de marques). C'est bien, de façon générale, un processus en jeu de miroir : «il s'agit de proposer, en tant que fabricant, sachant ce que pensent les clients de la marque ou de la combinaison de marques proposée ».

Pour y parvenir, nous évoquerons d'abord ( $c f$. 3.1.) la question centrale de l'incertitude puis ( $c f .3 .2$.) celle de l'application de la théorie du jugement.

6. C'est-à-dire la capacité effective, à un moment donné, à se déployer à nouveau sur un autre objectif précis : en anticipant ou par simple rebondissement. Pour une présentation élargie du problème à l'analyse du changement intra- et interorganisationnel, $c f$. BouvierPatron (2001).

Revue internationale P.M.E., vol. 16, n 1,2003 


\subsection{L'incertitude et la marque}

Le client (entreprise ou consommateur) veut être satisfait de son achat. Il éprouve donc le besoin, a priori, d'être rassuré. C'est pour cela que, dans sa quête des signaux lui donnant confiance, la marque joue un rôle si important.

On peut discuter longuement du rôle joué par les experts pour rationaliser et objectiver l'acte d'achat, et ce, y compris en « $\mathrm{B}$ toB $»$ puisque l'expert (acheteur) lui-même est « informé », donc sensible aux signaux et à la mode. Il se voit même interpellé par les marques qui vont communiquer en sa direction: l'objectif est, clairement, d'influencer les experts. C'est particulièrement vrai en direction des distributeurs, car le consommateur est attentif à l'avis des experts que sont les vendeurs : il s'agit donc ici de manipuler, indirectement, le consommateur en manipulant les experts. C'est une possibilité parmi d'autres : ainsi existent des solutions beaucoup plus ambitieuses lorsque l'entreprise détenant ou créant une marque d'ingrédient ( $c f . i$ ) supra) persuade les consommateurs finaux d'exiger la présence de l'ingrédient dans le produit acheté.

Le client, désireux d'être rassuré, reçoit des signaux (directement mais aussi indirectement avec d'autres signes officiels d'origine institutionnelle : la marque NF par exemple). Ces signaux visent, a priori, à donner confiance au client dans son acte d'achat, mais ils sont surtout destinés à garantir des débouchés : les entreprises utilisent donc volontiers ce vecteur. Ce qui peut alors poser problème aux clients est, finalement, plus la profusion de signaux que leur rareté ! Dans des cas extrêmes, les entreprises peuvent s'impliquer dans des stratégies d'image indépendamment, parfois, des caractéristiques réelles des produits. Il s'agit là, sciemment, de chercher à leurrer les clients. De telles stratégies misant sur la profusion de signaux, pour opérer une confusion dans l'esprit des acheteurs, visent à «faire des coups » commerciaux à court terme et de façon opportuniste. Le risque est, naturellement, de jeter le discrédit définitif sur le produit concerné ou une classe de produits ( $c f$. Akerlof, 1970). Néanmoins, de telles stratégies de prédation d'affaires tendent, a contrario, à long terme, à renforcer la logique fondamentale de la marque : la véritable marque étant un refuge sécurisant qui élimine la question de l'incertitude sur la qualité.

D'ailleurs, tant qu'il y a insécurité du consommateur et, donc, prédominance du subjectif, la notoriété de la marque est un refuge (signal de qualité). A contrario, lorsque l'insécurité (donc la subjectivité) disparaît, l'indicateur universel que constitue la marque tend à s'effondrer.

On sait que la marque, une fois reconnue, renferme un capital confiance sur lequel se fonde le jugement. Ce qui est en jeu est donc bien de déterminer les raisons pour lesquelles telle marque $\mathrm{X}$ d'ingrédient est appréciée par ses clients (cf. supra, Malaval, 1999). 
Plus précisément, pour nous, il s'agira surtout d'envisager une compréhension dynamique du phénomène de recours aux marques d'ingrédients ainsi que des logiques de combinaisons de marques (que nous résumons par l'expression «marque dans la marque »).

\subsection{La théorie du jugement étendue et appliquée à la marque dans la marque}

Dans le processus qui conduit à l'achat, l'acheteur (entreprise ou particulier) passe d'abord par une étape d'évaluation des solutions offertes pour satisfaire son besoin. Dans cette étape, pour élaborer son jugement (pour une présentation de ce point, $c f$. Lesavre, 1994, par exemple), l'acheteur attribue des performances aux offres (à partir de critères liés à des attributs donnés) en utilisant son expérience du critère et/ou des croyances issues de son environnement personnel, social, culturel et des stimuli commerciaux (venant ou non d'experts). Dans ce processus, la marque intervient comme un signal chargé de sens en fonction de la valeur de la marque. Cette valeur se résume dans un schéma (schéma 1) inspiré de Aaker (1991).

SCHÉMA 1

La qualité perçue selon Aaker

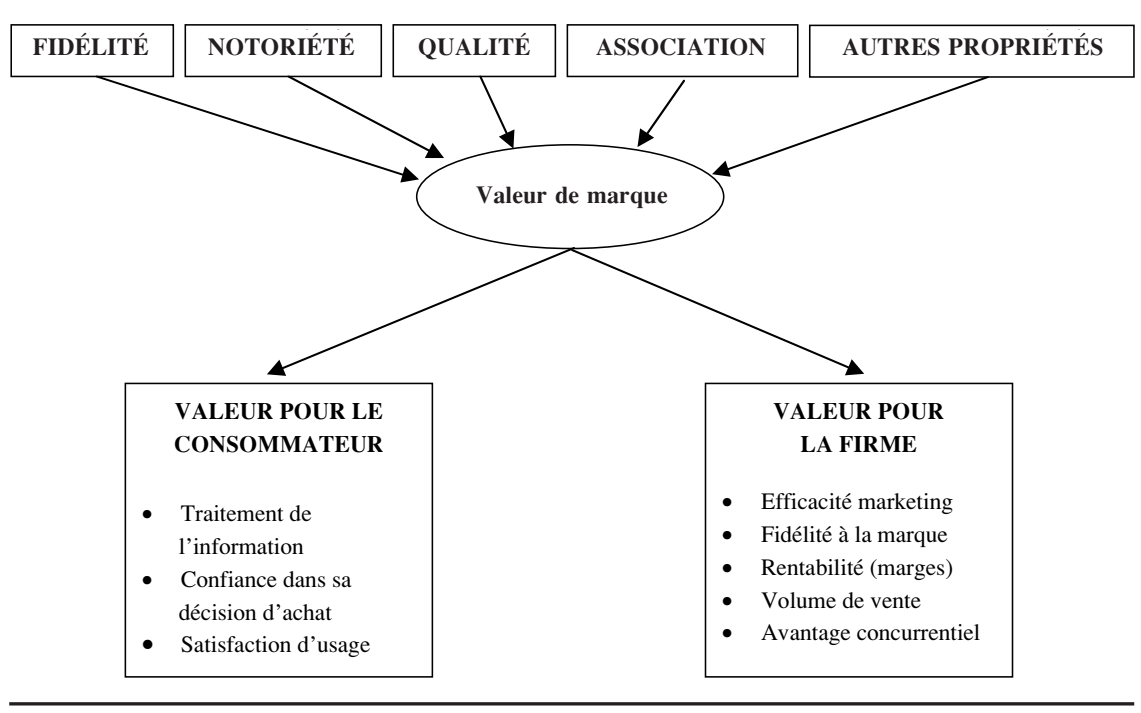

Dans notre optique, nous devons nous intéresser aux phénomènes de notoriété, de qualité perçue et d'associations. Ainsi, ici, les facteurs clés de succès s'appuient sur une construction de notoriété : ce qui est vrai pour toutes les marques.

Revue internationale P.M.E., vol. 16, nº 1, 2003 
En revanche, les marques qui nous intéressent semblent développer, dans l'esprit du client (entreprise ou consommateur) des phénomènes d'associations liés à la qualité perçue. Dans les composantes de la qualité perçue, Aaker (1991) distingue le contexte produit et le contexte service; nous nous attachons au contexte produit pour rester cohérent avec notre investigation.

La qualité perçue d'un produit, selon Aaker, est mesurée par le consommateur dans sept dimensions :

- performance fonctionnelle du produit (ce que le produit permet de faire): quoi / comment ;

- caractéristiques (selon les composantes du produit : p. ex., la présence du menthol dans une pastille assure au consommateur qu'il aura une bonne haleine);

- conformité aux attentes (ou, négativement, incidence des défauts sur la satisfaction);

- fiabilité du produit (conformité identique aux attentes en cas d'achat répété);

- durabilité (longévité de la fiabilité);

- serviabilité (qualité des services associés à l'acquisition et à l'usage du produit);

- adéquation globale (sentiment subjectif quant à l'aspect « coup-d' œil et sensation » inhérent au produit de qualité).

Ici, comme nous traitons de l'intégration d'autres marques (de type marques de composants ou, autrement appelées, marques d'ingrédients) dans la marque porteuse, l'objectif poursuivi par l'association est de parvenir à ce qu'un attribut de produit (lié à une caractéristique) soit synonyme du nom de marque de l'ingrédient : ainsi, p. ex., Lycra est devenu synonyme de brillance, élasticité et confort. Ce qui permet d'opérer une double réduction : réduction sémantique d'abord (lire le nom de la marque de l'ingrédient suffit à informer sur le produit) et réduction d'incertitude ensuite (rassurer le consommateur sur son acte d'achat).

Ainsi (phénomène d'association), la présence d'une marque d'ingrédient donnée fournit - à la marque porteuse (celle de l'ensemblier) - une performance sur une caractéristique précise et satisfaisant un besoin précis. En fait, l'acheteur va attribuer à la marque porteuse la performance de l'ingrédient sur une ou plusieurs caractéristiques.

Conformément à la théorie du jugement, le client va ainsi associer, par une croyance, la performance du produit de marque acheté à la présence de la marque d'ingrédient (dans la marque porteuse). La marque d'ingrédient agit comme un 
indicateur de performance de la marque porteuse sur un attribut donné alors que la preuve de la performance a été faite ailleurs et n'est pas observable directement par le client (au moment de l'achat en tout cas) tandis que le client fera bien l'expérience de cette performance après l'achat.

Ici aussi, en «BtoB », l'importance de la perception par le client ne saurait être démentie. La théorie du jugement est au fondement même de la formation de la qualité perçue du produit (de marque ou de marques) et s'appuie bien sur deux éléments fondamentaux :

- les croyances : ce que croit le consommateur ou ce à quoi il croit;

- l'expérience: confirmation apportée par l'usage sous forme de vérification; ce qui permet fidélisation du client et pérennisation du nom de marque.

Pour être schématique, on peut retenir à l'issue de ces deux sous-sections ( $c f$. 2.1. et 2.2. supra) deux éléments qui, à la base, constituent le socle sur lequel s'élaborent les facteurs clés de succès de la combinaison de marques :

- construire notoriété et réputation (c'est-à-dire faire en sorte qu'un ingrédient donné finisse par être synonyme d'un attribut donné);

- développer dans l'esprit des consommateurs/clients des phénomènes d'associations liés à la qualité perçue (c'est-à-dire présence de l'ingrédient de marque $\mathrm{X}$ dans la marque porteuse Y signifie l'existence d'une qualité perçue par le consommateur / client).

Bien entendu, ce qui peut être a priori (mais a priori seulement) perturbant est qu'un même ingrédient utilisé par deux ensembliers différents puisse conduire à deux discours différents (comme pour l'exemple de l'AntiBlockingSystem proposé par Kapferer (1998, p. 56) : «ABS chez Volvo => « sécurité »; ABS chez $\mathrm{BMW}=>$ «moyen d'aller plus vite » »). Il s'agit là simplement, outre la recherche d'un argument de vente pour le cœur de cible respectif de chaque constructeur automobile, de l'image institutionnelle (corporate) que veut se donner chacune de ces entreprises. Pourtant, il existe aussi ici un risque pour le fabricant de l'ingrédient (fournisseur du constructeur) s'il n'y prend garde : la banalisation. En effet, une forme de polysémie se développe et ceci est d'autant plus vrai que l'ingrédient considéré devient une marque générique: donc très diffusée mais aussi très banalisée ( $c f$. Swiners, 1979). Dans ce cas, il n'est pas certain pour autant que la marque d'ingrédient concernée résiste à cette diffusion: soit qu'elle ne puisse répondre à la demande, soit qu'elle succombe à une croissance trop rapide et mal maîtrisée, soit encore qu'elle se fasse imiter puis dépasser technologiquement. Ainsi, la diffusion sans frais de communication est une chose pouvant être a priori positive, mais la banalisation qui peut en résulter, pour un ingrédient très désiré, est un danger considérable pour la maîtrise de l'image et le maintien (ou la

Revue internationale P.M.E., vol. 16, n ${ }^{\circ} 1,2003$ 
croissance) de la position concurrentielle du fabricant d'ingrédient. Avant de diffuser, il faut donc que le fabricant-promoteur d'ingrédient s'assure, au préalable, d'un potentiel de croissance, d'une capacité à suivre une évolution rapide et dispose d'un souci de développement et d'innovation permanents des produits tout en négociant des liens privilégiés (de plus ou moins long terme) avec certains clients mais sans jamais oublier de prospecter pour élargir les débouchés. Aussi, en BtoB, un lien spécifique entre fournisseur et client (désireux d'élaborer un produit) débouche généralement sur des actions maîtrisées dans l'optique de capturer une clientèle propre : ce qui signifie, en amont, la construction possible d'une stratégie industrielle (choix de classe 2).

N'oublions pas également que, dans le processus d'achat, parmi les facteurs influençant le mode d'évaluation du produit, la peur (liée à un risque perçu dans l'acte d'achat) est déterminante. C'est pourquoi la possibilité d'un recours aux signaux simples que sont les marques joue un si grand rôle. Sans entrer dans le détail du concept de risque en marketing, celui-ci croît avec le degré d'implication dans l'achat ainsi qu'avec la faible compétence du client à évaluer une caractéristique donnée. Ainsi, dans l'exemple des ordinateurs personnels, les nouveaux acquéreurs sans connaissances informatiques vont se référer à un expert, le vendeur d'une enseigne de distribution ; lequel a aussi ses a priori et écoute la mode lorsqu'il n'a pas aussi été formé par le fabricant d'ingrédient. Il y a ainsi formation d'un processus de focalisation, à un moment donné : c'est le cas pour le microprocesseur Pentium d'Intel parce que symbolisant a priori une meilleure performance (en termes de puissance et de rapidité). Ce qui rassure le client (lequel connaît déjà la notoriété d'Intel) est de voir un vendeur (expert) lui confirmer l'association qu'il a déjà vu suggérer ailleurs (par la publicité notamment). De plus, l'industrielpromoteur (Intel) a, avec les incorporateurs-ensembliers (fabricants de PC), parfaitement coordonné, par des budgets marketing, les actions suscitant un lien spécifique avec l'aval (clients finaux) : «Intel Inside » en est l'illustration. Au total, tout en étant en BtoB, Intel combine l'action poussée en s'appuyant sur les distributeurs (dont les vendeurs ont été formés, pour tout ou partie, par l'industriel-promoteur) et l'action tirée de sorte à toucher directement les clients finaux. Tous les interlocuteurs rassurés, le risque est perçu moindre pour l'ensemble de la filière.

\section{Conclusion}

\section{Guide d'usage de la marque dans la marque (croisement Stratégies et FCS)}

La synthèse issue de notre investigation peut être désormais effectuée.

Nous avons cherché à incorporer à l'analyse les points de vue des acteurs concernés (fabricants de marques d'ingrédients et incorporateurs de marques 
d'ingrédients) puis nous avons proposé les stratégies (impliquant, en particulier, des combinaisons de marque avec d'autres du fait de complémentarités verticales) et les facteurs clés de succès.

Les facteurs clés de succès (f.c.s. ou FCS) fondamentaux, donnant les conditions de base pour envisager des combinaisons de marques viables, sont les suivants :

i) orienter le canal de la consommation vers son produit par sa marque;

ii) être le premier entré ou être supposé tel, à condition toutefois de savoir construire une barrière à la mobilité pour préserver, sinon l'avantage concurrentiel initial, du moins une avance sur les suiveurs ${ }^{7}$ et / ou perçu comme tel par les clients potentiels, car la visibilité commerciale est essentielle ( $c f$. supra) : donc, un leadership avec une aptitude à préserver durablement l'avantage ;

iii) aptitude à la mise en place d'un ensemble de compétences donnant une capacité générique et rapidement redéployable (sur un nouvel enjeu technologique ou bien par des adaptations continues des ingrédients / prestations proposés aux clients): disposer de compétences donnant une capacité générique rapidement redéployable ;

iv) existence d'une complémentarité technique offrant des garanties crédibles pour une véritable stratégie industrielle commune permettant de constituer un avantage concurrentiel effectif ( $c f$. supra, classe 2): favoriser les complémentarités techniques.

Notons que ces différents facteurs peuvent être cumulatifs, généralement de $i v$ ) vers $i$ ), même si nous nous limiterons ( $c f$. tableau 2) à donner les exemples centrés sur chacun de ces niveaux.

Ce qui est ainsi recherché prioritairement par les entreprises est un double verrouillage :

- chercher à préempter, si possible, l'image d'un positionnement de leader historique (de façon à asseoir l'idée qu'une première marque sur un segment de marché, si elle s'avère satisfaisante ou réputée telle par les clients, devient la référence nominale, voire la référence tout simplement);

7. Cela dit, être suiveur innovant grâce aux compétences détenues et en cours d'appropriation, seul ou avec d'autres, permet de s'inscrire sur un segment (même faible initialement) et, par apprentissage (mais aussi observation du leader et d'autres suiveurs), d'obtenir un positionnement continûment amélioré (et parfois même à rejoindre et / ou dépasser le leader).

Revue internationale P.M.E., vol. 16, n ${ }^{\circ} 1,2003$ 
- mais aussi préempter le canal de diffusion lui-même (en orientant l'acte d'achat des consommateurs) afin qu'il y ait répercussion vers l'amont, à travers les distributeurs puis les producteurs, d'une demande exclusive sur un produit particulier ou une marque particulière.

Dès lors, la capacité de déployer rapidement des compétences sur un nouvel objectif est un atout évident: pour faire perdurer un avantage concurrentiel; pour se mobiliser sur un nouveau défi (corrélatif à un changement, plus ou moins brutal, des conditions économiques extérieures sur un segment de marché, un marché, une branche). Un des paris fondamentaux à réaliser est de disposer d'un savoir, d'un savoir-faire et de coûts moindres pour proposer des produits innovants de qualité. Cela passe par le développement d'une collaboration indispensable avec des spécialistes sur la base de complémentarités d'activités.

À cette logique volontariste des entreprises, il faut ajouter un verrouillage supplémentaire induit par le comportement des consommateurs eux-mêmes. Comportement qui, d'après Chetochine (1995), correspond à v) un sentiment d'incompétence du consommateur dans son acte d'achat l'obligeant, par peur induite et besoin de se rassurer, de passer par la marque qui restaure sa confiance et lui permet d'acheter. Cela joue toujours lorsqu'une ou plusieurs caractéristiques, voire le produit dans son ensemble, sont trop complexes à évaluer par le consommateur. C'est donc là un facteur clé de succès générique plaidant pour l'existence d'une marque ou d'un label et que nous ne retiendrons pas.

La bonne identification par le client va d'abord passer par une forte notoriété de marque; cependant, il ne s'agit pas d'un facteur clé de succès permettant, à chaque fois, au promoteur d'un produit donné ou d'une marque d'avoir l'assurance que le client va s'orienter sur sa marque particulière en excluant les autres. En fait, cela permet seulement de diminuer le risque commercial pour le fabricant et, en ce sens, il s'agit d'une clé de succès minimale en tant que simple modérateur de risque.

Par ailleurs, nous avons aussi identifié les stratégies possibles (dans un continuum large : à savoir, seulement sa marque seul et/ou avec d'autre(s) versus la marque commune :

a) défendre sa marque seul en n'utilisant que des ingrédients que l'on rend insignifiants ;

b) défendre sa marque tout en faisant appel à d'autres entreprises réputées qui travaillent «silencieusement» pour le compte de cette marque (soit comme sous-traitant, soit comme partenaires);

c) défendre sa marque en recourant explicitement à d'autres marques complémentaires;

d) défendre une marque commune de façon conjointe de la part d'entreprises ayant des activités similaires.

Revue internationale P.M.E., vol. 16, n 1, 2003 
Il s'agit là de situations pures, mais, à l'évidence, des solutions mixtes peuvent coexister (simultanément) et perdurer au sein du portefeuille fournisseurs du client dans le cadre de l'élaboration d'un produit ou des produits.

Par commodité, on présentera, au total, dans un tableau (tableau 2), les idéauxtypes (sous formes d'illustrations par des exemples statiques de grandes marques connues) résultant du croisement entre les stratégies possibles $(a, b, c, d)$ et les facteurs clés de succès $(i, i i, i i i, i v)$.

La perspective de réussite d'une combinaison de marques implique d'abord la définition claire des objectifs de chacun et d'un engagement interrelationnel adéquat. Dans un contexte concurrentiel de niches et une logique industrielle de collaboration bien comprise basée sur des complémentarités, il faut idéalement envisager une démarche partenariale de co-évolution telle qu'elle est donnée par le résultat du couple $(i v, c)$. Notons qu'il s'agit là d'une voie présentée comme idéale mais qui s'avère accessible. Parmi le spectre des différentes possibilités offertes (Bouvier-Patron, 1994, 1998a), la relation partenariale est un idéal-type essentiel pour entreprendre une démarche de marques combinées, mutuellement avantageuse et durable.

Plus largement, la PME (dotée d'un pouvoir de négociation grâce aux compétences détenues : Bouvier-Patron 1994, 1996, 1998a, 1998b) dispose, du fait de la complémentarité $i v)$, d'une panoplie stratégique $(a, b, c, d)$ soutenant l'expansion et le changement par la conquête d'autres niveaux $(i, i i, i i i)$. Observons d'ailleurs qu'en $i v$ (ligne $i v$ du tableau 2), l'angle adopté est celui des modalités relationnelles dans une acception générique afin de bien faire comprendre les enjeux véritables du débat, à savoir que la réussite aval de la marque, individuelle ou conjointe, passe par une structuration relationnelle de qualité en amont.

Autrement dit, un bon marketing aval ne peut rien sans un potentiel organisationnel amont performant, lequel permet de mobiliser les compétences complémentaires requises et, également, les meilleurs projets techniques. Du reste, la dynamique industrielle porteuse de réussite semble bien passer par des structures organisationnelles souples et adaptées qui favorisent l'émergence et la gestion des innovations, le développement des produits, les effets de réputation positive liés ; ce qui donne alors les conditions possibles de l'assise de la marque (et cela s'impose d'autant plus dans une démarche de marque combinée) indépendamment même du phénomène d'extension de marque (sur ce point, $c f$. Cegarra, 1994, et Kapferer, 1998).

Sur le plan conceptuel, la notion de «marque partagée » (Kotler et Dubois, 1997, p. 446), volontairement mise de côté car trop floue et qui a été traduite, dans la pratique, par une nouvelle forme de promotion, doit donc être soit abandonnée, soit reconsidérée dans l'optique soulignée par Heilbrunn (1997, p. 1998) à la suite

Revue internationale P.M.E., vol. 16, $\mathrm{n}^{\circ}$ 1, 2003 


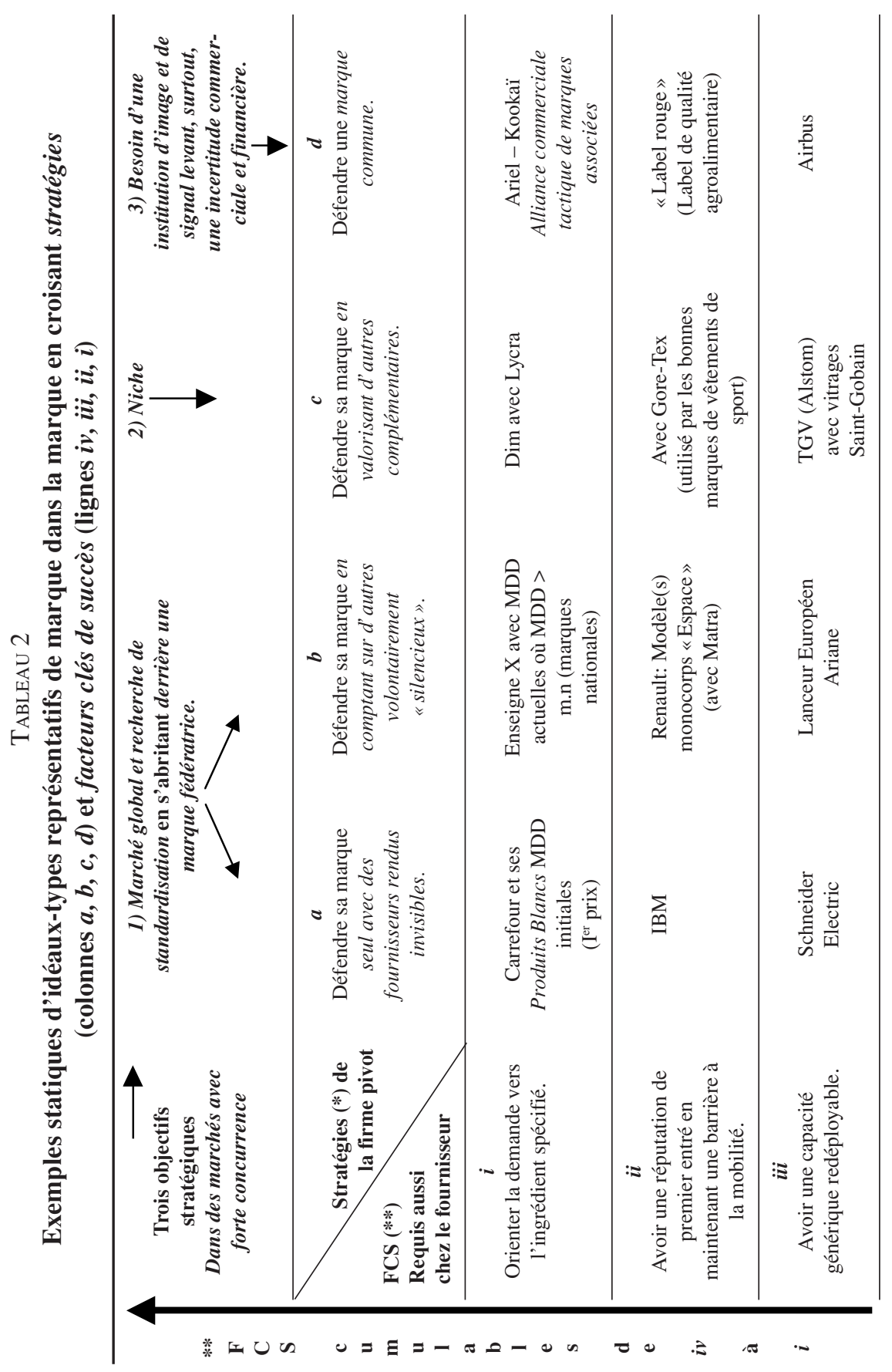

Revue internationale P.M.E., vol. 16, n 1,2003 


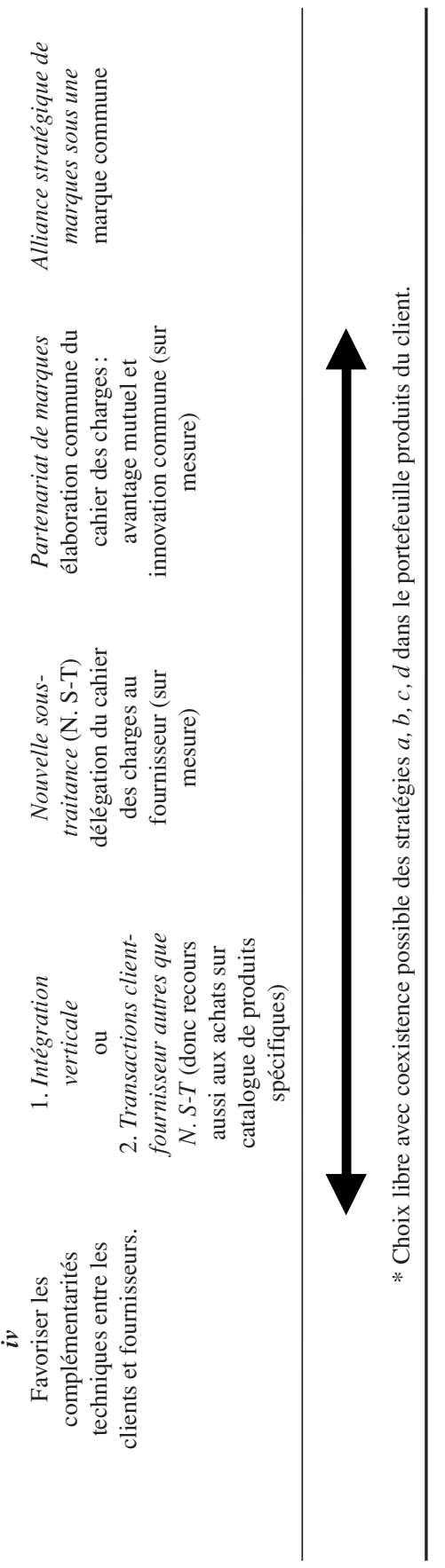

Revue internationale P.M.E., vol. 16, n 1,2003 
de Rao et Ruekert (1994). L'optique choisie ici est de proposer un angle d'analyse plus précis et plus systématique en éliminant toute connotation prétablie. Dès lors, partir, par exemple, de la notion d'alliance de marques est plus efficace. Toutefois, cette terminologie d'alliance est elle-même connotée. En outre, elle masquerait, par trop, les degrés possibles d'hybridation subtils du continuum allant de la marque individuelle à l'alliance de marques de type marque commune. Nous avons donc préféré utiliser le concept de marque dans la marque pour explorer une réalité complexe, mais fondamentale dans le cadre du fonctionnement en «métaorganisation » étant donné le développement des relations interentreprises.

En poussant la réflexion à l'extrême de manière à valider l'optique générale de la combinaison de marques (articulation et/ou coexistence de marques plutôt que leur suppression), il est intéressant de s'appuyer sur l'analyse de l'avenir d' une marque en cas d'acquisition: au sens où, selon le principe «qui peut le plus, peut le moins », la résistance de la marque à une logique de droits de propriété doit être envisagée comme un indice de robustesse de la réflexion proposée ici. Ainsi, le travail de Paturel et Thévenard (1999) ${ }^{8}$ montre plusieurs choses essentielles et interprétables favorablement pour la coexistence de marques. Trois constats s'imposent: dans le cadre des opérations de croissance externe, les marques cibles (à savoir celles de l'entreprise dont on prend le contrôle) ont, a priori et sans aucun déterminisme, autant de chances d'être conservées que supprimées; ce sont, en fait, surtout les difficultés d'intégration qui conduisent, à terme, à des suppressions de marque; plus la notoriété est grande, plus la probabilité de conservation d'une marque est élevée. Si l'étude réalisée ne montre pas de différence entre le sort des marques ressources (orientation marketing) et celui des marques compétences (réseau de compétences), c'est en grande partie parce que l'étude privilégie les produits de consommation finale.

Au total, l'intérêt intrinsèque de négliger la potentialité d'une combinaison de marques ou bien, très radicalement, l'acte habituel de faire complètement disparaître une marque d'ingrédient existante au profit de la seule marque du coordinateur (firme pivot) est généralement discutable, voire un fait absolument contre-productif ${ }^{9}$ en BtoB. Pour le comprendre et déterminer l'issue souhaitable au cas par cas, seule va importer l'analyse du contexte concurrentiel ( $c f$. bord supérieur du tableau 2)

8 L'optique développée par les auteurs est de proposer un guide aux dirigeants leur permettant d'anticiper le devenir d'une marque acquise.

9. Cette contre-productivité existe sauf dans une logique de domination push compréhensible par la nécessité d'accéder plus facilement, ainsi que d'être mieux perçu sur le marché aval. Or, cette idée très cohérente avec des marchés de masse, sauf exception, est assez peu compatible avec le BtoB où les marchés de niches sont déterminants et où les barrières sont multiples.

Revue internationale P.M.E., vol. 16, nº 1, 2003 
ou, plus exactement, la recherche d'adéquation entre la nature concurrentielle d'un marché donné et le positionnement stratégique (principe de bonne adéquation) plutôt que, par exemple, l'analyse de la concurrence ou du nombre de concurrents.

En synthétisant la totalité de nos propos, il s'agit alors bien d'articuler plusieurs niveaux :

- L'assise d'une entreprise sur un cœur de métier implique, à terme, que cette entreprise communique par le biais d'une marque si, toutefois, le souhait est d'accroître la part de marché, sortir de la niche d'origine et perdurer.

- L'usage de cette marque supportant un ingrédient explicite (plutôt que la dissimulation de ce dernier), dans la chaîne de valeur complexe de coordination du produit (initiée par la firme pivot du réseau de compétences), peut être envisagé comme une garantie en même temps qu'une chance de valorisation supplémentaire du produit final.

- Une politique de combinaison de marques peut alors être mise en œuvre mais, alors, en s'aidant d'instruments de contrôle.

Sur ce dernier point en effet, à titre d'exemple, la voie idéale du partenariat (et du partenariat de marques) déjà citée ne se décrète pas et exige, si on l'envisage comme norme à atteindre, des règles autorisant un engagement et assurant un contrôle pour indiquer ou anticiper toute possibilité de dérive immédiate ou ultérieure. Deux phases importent alors : A. le contrôle ex ante avant engagement; B. le contrôle in situ pendant la relation.

L'optique adoptée ici vise à guider le décideur en limitant les erreurs d'action possibles sans nécessairement garantir le succès.

A. Pour l'engagement, celui-ci est autorisé en vue d'une bonne probabilité de succès s'il y a vérification simultanée de trois critères concernant la relation à nouer entre deux partenaires potentiels :

- complémentarités de compétences et/ou d'activités ;

- symétrie relationnelle (donc pouvoir de négociation équilibré);

- élaboration d'un projet et d'un objectif commun à moyen ou long terme.

Ainsi, a contrario, ne pas s'engager (infirmation des trois critères) revient à ne pas démarrer une opération, a priori, préjudiciable pour l'une et / ou l'autre des parties.

B. Après l'engagement, pour le suivi de la relation, des indicateurs quantitatifs et qualitatifs, de nature interne et externe, vont d'abord permettre de valider le plus ou moins grand succès (le sens de variation positif de chaque indicateur 
sanctionnant positivement la politique conduite) et d'anticiper (donc de corriger) tout dérapage éventuel (lequel sera hypothétique une fois l'engagement effectué avec la garantie donnée par vérification des trois critères, $c f$. supra).

Indicateurs externes mesurant les retours attendus de la relation engagée sur une période :

- hausse de la fréquence des commandes émanant du portefeuille clients déjà constitué;

- hausse du nombre de clients par niche ciblée (donc niches initiale et connexes);

- aptitude permise à pénétrer de nouvelles niches depuis l'engagement.

Indicateurs internes du bon fonctionnement d'une interrelation partenariale :

- qualité croissante de la relation (collaboration satisfaisante, apprentissage, avantage mutuel, échanges réciproques, confiance, stabilisation relationnelle);

- répartition efficiente des tâches;

- maintien des identités respectives;

- renforcement des capacités respectives (financières, humaines, organisationnelles, technologiques et innovationnelles).

La mise en œuvre des critères ex ante et des indicateurs in situ offre aux entreprises en $\mathrm{B}$ toB un instrument de contrôle et de vérification encore imparfait mais à la fois global et applicable pour le lancement éventuel, l'engagement, la mise en place et la surveillance d'un projet de marque combinée (parmi les possibilités explorées à l'aide du tableau 2).

Ainsi, le coeur de compétences de chaque entreprise est un élément moteur de rétablissement d'équilibre au sein des relations clients-fournisseurs (lesquelles sont a priori asymétriques du fait de la taille).

De plus, selon les modalités contractuelles, la marque peut jouer un rôle essentiel de consolidation par rapport à l' excellence acquise par les compétences (ce qui permet d'asseoir, plus largement et plus durablement, un ré-équilibre des relations); on passe alors par des combinaisons de marques selon l'éventail des possibles; combinaisons dont la mise en œuvre effective et le succès dépendent du respect de modalités de contrôle. 


\section{Bibliographie}

AAKER, D. (1991), Managing Brand Equity, New York, The Free Press.

AOKI, M. (1988), Information, Incentives and Bargaining in the Japanese Economy, Aoki, Masahiko Cambridge, Cambridge University Press.

ASANUMA, B. (1989), « Manufacturer-supplier relationships in Japan and the concept of relationspecific skill », Journal of the Japanese and International Economies, p. 1-30.

AXElRod, R. (1984), The Evolution of Cooperation, New York, Basic Books.

AKERLOF, G. (1970), «The market for "Lemons": quality uncertainty and the market mechanism », Quarterly Journal of Economics, p. 488-500.

Bouvier-PATRON, P. (1994), «La dimension inter-entreprises de production dans le modèle "J" d'Aoki », Revue d'économie politique, n' 1, p. 120-144.

BOUVIER-PATRON, P. (1996), «Pouvoir et/ou taille de la firme : quelle dimension pertinente pour analyser les relations interentreprises? », Sciences de la société, $\mathrm{n}^{\circ} 38$, p. 49-64.

BOUVIER-PATRon, P. (1998a), «Stratégies relationnelles entre les enseignes de grande distribution et leurs fournisseurs », Économie rurale, $\mathrm{n}^{\text {os }} 245-246$, p. 69-74.

Bouvier-PATRON, P. (1998b), «Coordination et intelligence relationnelle au sein de l'organisation industrielle », Revue d' intelligence économique, $\mathrm{n}^{\circ} 3$, p. 12-26.

BOUVIER-PATRON, P. (2001), «Analysis of change within and between firms embedded in their environment in the context of increasing interactions : understanding complexity of organizations », European Journal of Economic and Social Systems, introduction (p. 1-38), numéro spécial «Analysis of change within and between firms ».

CAVES, R.E. et M.E. PORTER (1977), « From entry barriers to mobility barriers : conjectural decisions and contrived deterrence to new competition », Quarterly Journal of Economics, p. 241-261.

Cegarra, J.J. (1994), «Gérer un portefeuille de marques », Décisions Marketing, no 3, p. 81-91.

Chetochine, G. (1995), La déroute des marques, Paris, Éditions Liaisons.

HeIlbrunn, B. (1997), «La Marque», dans Encyclopédie de Gestion, Y. Simon et P. Joffre (dir.), 2e édition, Paris, Economica, p. 1973-2007.

KAPFERER, J.N. (1998), Les Marques : capital de l'entreprise, 3 e édition Paris, Éditions d'Organisations.

KAPFerer, J.N. (2000), Remarques, Paris, Éditions d'Organisations.

KNight, F. (1921), Risk, Uncertainty and Profit, Chicago, The Riverside Press Cambridge.

Kotler, P. et B. Dubois (1997), Marketing Management, 9e édition, Paris, Publi-Union.

KREPS, D. et R. WILSON (1982), «Reputation and imperfect information », Journal of Economic Theory, vol. 27, p. 253-279.

Revue internationale P.M.E., vol. 16, nº 1, 2003 
LESAVRE, L. (1994), «Perceived quality in industrial buying: a conceptual approach », dans Perspectives on Marketing Management, vol. 4, p. 275-291, John Wiley and Sons.

Malaval, P. (1996), Marketing Business to Business, Paris, Publi-Union.

MalaVal, P. (1998), Stratégie et gestion de la marque industrielle, Paris, Publi-Union.

Malaval, P. (1999), «Typologie des marques industrielles », Revue française de marketing, $\mathrm{n}^{\text {os }} 173-174$, p. $71-85$.

MAlaval, P. et C. BÉnAROYA (1998), «Les marques de distributeurs dans le contexte industriel », Décisions Marketing, no 15, p. 59-68.

PATUREL, R. et C. THÉVENARD (1999), «Le sort des marques acquises lors des opérations de croissance externe », Finance Contrôle Stratégie, vol. 2, nº 2, juin, p. 159-190.

Porter, M. (1980), Competitive Strategy, New York, The Free Press.

Porter, M. (1985), Competitive Advantage, New York, The Free Press.

RICHARDSON, G.B. (1972), «The organization of industry », Economic Journal, p. 883-896.

RAO, A.R. et R.W. RUECKERT (1994), «Brand alliances as signals of product quality », Sloan Management Review, p. 87-97, Article traduit dans R.W. Rueckert, A.R. Rao et C. Benavent (1994), «Alliances de Marques », Décisions Marketing, n 1.

SCHELling, T. (1956), «An essay on bargaining », American Economic Review, p. 283-306.

SCHELling, T. (1960), The Strategy of Conflict, Cambridge, Harvard University Press.

SELten, R. (1978), «The chain store paradox », Theory and Decision, dans R. Selten (1988), Models of Strategic Rationality, Dordrecht, Kluwer Academic Publishers.

SWINERS, J.L. (1979), «Bilan critique du rôle de la copy stratégie dans la pratique publicitaire actuelle », IREP, juin, 19e journée d'études, Paris.

TEECE, D.J. (1980), «Economies of scope and the scope of the enterprise », Journal of Economic Behavior and Organization, p. 223-247.

TEECE, D.J. (1988), « Technological change and the nature of the firm », dans G. Dosi et al., Technical Change and Economic Theory, Londres, Pinter Publishers, p. 256-281. 